a un inconvenient, celui que la pression de la roue d'echappement se fait contre la longueur de la détenteressort; ils craignent que le ressort de la détente, étant extrêmement faible, n'ait pas une assiette aussi fixe que dans lechappement d'Arnold, ou la pression de la roue d'echappement se fait suivant la longueur du ressort et où la roue tend visiblement à maintenir la détente daus une position conslamment égale. Quoique la bonne marche de bien des horloges, pourvues de lechappement d'Earnshaw, semble prouver que cette crainte n'est pas bien fondée, il est cerlain qu'il y a quelque chose qui fera que sous ce rapport plusieurs donneront encore la préférence à l'echappement d'Arnold, surtout depuis le moment qu'il a donné plus de diamètre à son cercle d'echappement qu'il ne faisait auparavant. Je n'entre point dans les discussions qui ont

Copenhague, 30 May 1822. eu lieu à cet-egard, mais ce que je crois ponvoir assurer, c'est que par la disposition de l'echappiment que j'ai proposé, le défaut que plusieurs ont reproché à l'cchappement d'Larnshaw, s'il existe effectivement, se trouvera reduit à la moilié, et cela puiceque la roue d'arrêt n'agit qu'avec la moitié de la force environ, de celle de l'echappement avec une senle roue, el c'est par colte force on pression que la détente flécherait, si toute fois elle fléchit pendant le jeu de l'echappement.

Les remarques peu favorables d'un arliste si celèbre que Mr. Earnshaw, ne sauront m'empêcher de faire usage de mon echappement, et si contre mon attenle j'y découvre des inconveniens, j'aurai la bonne fui de les publier moi - même.

\section{Urban Jürgensen.}

\title{
Erreur à la gravure représentant l'echappement libre a double roue.
}

Les dents de la petite roue d'echappenent ou celle d'impulsion ne sont pas représentécs tout à fait à la gravure comme elles le sont au modèle, ou leur forme est à peu près comme celle des dents d'une roue de rencontre ordinaire, c'est-à-dire assez inclinées pour que la pointe des dents agisse seule sur la palette ou l'encoche du cercle d'echappement. Cette petite erreur du gravenr n'empêchera au resie pas de comprendre également bien le jeu de l'echappement.

\section{Auszug aus einem Briefe des Herrn Professor Wurm an den Herausgeber:}

Aus älteren Beobachtungen von 1791 bis 1799 erhielt ich. die Länge von Hamburg zwischen $29^{\prime} 57^{\prime \prime}, 3$ und $30^{\prime} 18^{\prime \prime}, 0$ östlich in Zeit von Paris. Spätere Beobachtungen von 1800 bis 1803 gaben mir diese Länge um ein beträchtliches grösser, lassen sich aber, da nicht alle an demselben Orte in Hamburg gemacht wurden, nicht gradezu mit einander vergleichen. Was für eine Lünge jede dieser Beobachtungen von 1791 an besonders giebt, findet sich in meinen zwei alphabetischen, isn $2^{\text {ten }}$ und $26^{\text {sten }}$ Bande der M. C. abgedruckten Längenverzeichnissen. Ich begnüge mich hier blos die neueren von mir berechneten Beobachtungen seit 1806 anzufüliren.

A) Repsolds Beobachtungen auf sciner vorigen Sternwarte

$$
\begin{array}{llll}
\text { 1806 } & \text { 16. Jan. } & \text { Cfinsternifs } & 3^{\prime} 34,6 \\
\text { 1808 } & \text { 31. März. } & \text { W Stier } & -25,3 \\
\text { 1810 } & \text { 18. Scpt. } & \text { Aldebaran } & -32,6
\end{array}
$$

B) Rümkers Beobachtungen auf seiner Sternwarte. (Reduction auf Michaelis $+\mathrm{I}^{\prime \prime} .0$ )

$$
\begin{array}{rll}
\text { I820 23. April. } & \chi \text { Löwe } & 30^{\prime} 28,7 \\
\text { 21. Mai. } & 89 \text { Löwe } & -35,3 \\
\text { 29. Aug. } & \text { Alcyone } & -11,9 \\
\text { 29. Aug. } & \text { Merope } & -28,9 \\
\text { 7. Sept. } & \text { Cfinsternifs } & -38, \mathbf{I} \\
\text { 1821 } & \text { 9. Febr. Taygeta } & -36,3 \\
\text { 9. Febr. } & \text { Maja } & -37, \mathbf{I} \\
\text { 9. Febr. Asterope k } & -41,4 \\
\text { 9. Febr. } & \text { Celreno } & -31,2
\end{array}
$$

Aus diesen Beobachtungen fulgt mit Ausschlurs von Alcyone die Länge von Rä̈nker's Sternwarte im Mittel 
$30^{\prime} 34^{\prime \prime}, 6$ und damit die Länge des Michaelisthnrms in Hamburg

$$
30^{\prime} 35^{\prime \prime}, 6 \text { *) }
$$

Die Länge von Frederiksvärk auf Seeland habe ich aus dem daselbst beobachteten Ende der Ofinsternifs rom 7. Sept. $1820 \mathrm{zu} 38^{\prime} 54^{\prime \prime}, 3$ in Zeit von Paris berechnet.

\section{Wurm.}

*) Meine. Wohnung in Altona liegt nach eine vorläufige Verbindung, $8 \prime \prime, 6$ in Zeit, westlich vom Michaelis, und in $53^{\circ} 32 \prime 51 /, 3$ der Breite.

\section{Bemerkungen über barometrische $\mathrm{Nivellements.}$ Von M. Navier.}

(Aus Annales de Chemie et de Physique. T. XIX. Janvier 1822.)

Wenn man die Veränderung der Schwere in der verticalen Richtung ansser Acht lärst, so ist die Formel, mittelst welcher man aus Barometerbeobachtungen Höhen berechnet, diese

$$
Z=A(1+0,002 \nu) \cdot\left(\log \frac{H}{h}+0,00007825 u\right)
$$

wo $\boldsymbol{Z}$ den gesuchten Höhenunterschied bezejchnet; $A$ einen numerischen Coefficienten, der für den mittlern Parallelkreis $=18393 \mathrm{~m}$ ist, und mit der Breite sich nach einem bekannten Gesetze veründert; $v$ die Summe der Lufttemperaturen in beyden Stationen; $\boldsymbol{H}, h$ die beobachteten Barometerhöhen resp. in der untern und öbern Station; $u$ die Temperatur im öbern Standpunkt, wenn die im untern davon abgezogen. Die Zahl 0,00007825 ist das Product der cubischen Ausdehnung des Quecksilbers, 5550, mit 0,434295 als dem Verhältnifs der gewöhnlichen zu den hyperbolischen Logarithmen.

Wenn man auf die Ausdehnung der Barometerscale Rücksicht nimmt, murs man die Linearausdehnung der Materie, woraus solche besteht, zu der cubischen des Quecksilbers legen. Die bekannten Resultate hier angewandt, geben den Coefficienten von u für Scalen von Glas oder Holz 0,00008505, und fiir messingene 0,00008641 , welche Verbesserung nicht vernachlässigt werden darf.

Man hat Tafeln, welche für jede Breite den Logarithmus von $A$ gehen. Auch kann man zum voraus die Producte des Coefficienten von $u$ mit den ganzen Zahlen von 1 bis 9 bilden. Durch diese Hülfsmittel scheint die Berechnung von $Z$ nach obiger Formel eben so leicht zu werden, als der Gebrauch der verschiedenen Tafeln, die sonst zur Exleichterung dieser Operation eingerichtet sind.

Wenn eine Gröfse, so wie hier, aus mehreren, durch Beobachtungen gegebenen, Elementen gefunden wird, so ist solche einem Fehler unterworfen, der von den in den einzelnen Elementen begangenen Fehlern abhängt. Es ist sehr nützlich zu wissen, welchen Einflufs aufs Ganze ein Fehler in jedem einzelnen Element habe. Man kann alsdenn von dem möglichen Grade der Annäherung des Ganzen urtheilen; und weifs, welche Elemente es vorzïglich wichtig ist, mit Genauigkeit zu erhalten.
Betracliten wir eine Function $U$ ron verschiedenen veränderlichen Gröfsen $x, y$ etc. Wenn der Werth von $x$ um eine sehr kleine Grölse $\Delta_{x}$ vermehrt wird, so ist, wie man aus den Grundsätzen der Differenzialrechnung weifs, die daraus folgende Vermehrung von $U$ sehr nahe

$$
\frac{d U}{d x} \cdot \Delta x
$$

Folglich werden, wenn wir mit $\Delta x, \Delta y$ etc. die kleinen in den Elementen $x, y$ etc. begangenen Fehler bezeichnen, die Einflüsse derselben, auf die Funkion $U$

$$
\frac{d U}{d x} \cdot \Delta x, \frac{d U}{d y} \Delta y
$$

und die relativen Fehler, das heifst die Verhältnisse der Fehler in $U$ gegen den Werth dieser Funktion, sind

$$
\frac{1}{U}\left(\frac{d U}{d x} \cdot \Delta x+\frac{d U}{d y} \cdot \Delta y+\text { etc. }\right) \text {. }
$$

Indem wir, dies auf die oben angeführte Formel anwenden, finden wir

1) dars der Fehler $\Delta_{\nu}$ in der Summe der Lufttemperaturen, in $Z$ einen relativen Fehler verursache von

$$
\frac{0,002}{1+0,002 v} \cdot \Delta v
$$

2) Dafs den Fehlern $\Delta H, \Delta h$ in den Barometerhöhen, folgende relativen Fehler in $Z$ entsprechen

$\frac{N}{\log \frac{H}{h}+0,00007825 u} \cdot \frac{\Delta H}{H}$, und $\frac{-N}{\log \frac{H}{h}+0,00007825 u} \cdot \frac{\Delta h}{h}$

$N$ bedeutet die Zahl 0,434295 .

3) Dafs der durch den Fehler $\Delta^{u}$ in der Differenz der Barometertemperaturen entstandene relative Fehler in $U$ dieser. sey

$$
\frac{0,00007825}{\log \frac{H}{h}+0,00007825 u} \cdot \Delta u
$$

Aus diesen Resultaten zielen wir folgende allgemeine Schliüse :

1) Der Einflufs eines Fehlers in den Lufttemperaturen hängt von der 211 messenden Höhe nicht ab. Dieser Einflufs ist 